Original article

\title{
Routine transthoracic echocardiography in a general Intensive Care Unit: An 18 month survey in 704 patients
}

\author{
Paulo Alexandre Marcelino*, Susan Marques Marum, Ana Paula Matos Fernandes, \\ Nuno Germano, Mario G. Lopes \\ Intensive Care Unit, Hospital Curry Cabral, Lisbon, Portugal
}

Received 29 September 2007; accepted 24 September 2008

Available online 20 November 2008

\begin{abstract}
The authors analyzed 704 transthoracic echocardiographic (TTE) examinations, performed routinely to all admitted patients to a general 16bed Intensive Care Unit (ICU) during an 18-month period. Data acquisition and prevalence of abnormalities of cardiac structures and function were assessed, as well as the new, previously unknown severe diagnoses.

A TTE was performed within the first $24 \mathrm{~h}$ of admission on 704 consecutive patients, with a mean age of $61.5 \pm 17.5$ years, ICU stay of $10.6 \pm$ 17.1 days, APACHE II 22.6 \pm 8.9 , and SAPS II 52.7 \pm 20.4 . In four patients, TTE could not be performed. Left ventricular (LV) dimensions were quantified in $689(97.8 \%)$ patients, and LV function in $670(95.2 \%)$ patients. Cardiac output (CO) was determined in $610(86.7 \%)$, and mitral $E / A$ in 399 (85.9\% of patients in sinus rhythm). Echocardiographic abnormalities were detected in 234 (33\%) patients, the most common being left atrial (LA) enlargement $(n=163)$, and LV dysfunction $(n=132)$. Patients with these alterations were older $(66 \pm 16.5$ vs $58.1 \pm 17.4, p<0.001)$, presented a higher APACHE II score $(24.4 \pm 8.7$ vs $21.1 \pm 8.9, p<0.001)$, and had a higher mortality rate $(40.1 \%$ vs $25.4 \%, p<0.001)$. Severe, previously unknown echocardiographic diagnoses were detected in 53 (7.5\%) patients; the most frequent condition was severe LV dysfunction. Through a multivariate logistic regression analysis, it was determined that mortality was affected by tricuspid regurgitation ( $p=0.016$, CI $1.007-$ 1.016) and ICU stay $(p<0.001$, CI 1-1.019). We conclude that TTE can detect most cardiac structures in a general ICU. One-third of the patients studied presented cardiac structural or functional alterations and 7.5\% severe previously unknown diagnoses.
\end{abstract}

(C) 2008 European Federation of Internal Medicine. Published by Elsevier B.V. All rights reserved.

Keywords: Transthoracic echocardiography; Intensive care; Data acquisition

\section{Introduction}

Echocardiography is a promising technique in several noncardiological settings, such as general Intensive Care Units (ICU) or emergency departments. It is a sensitive tool used to detect the presence of specific cardiovascular abnormalities, which may be life threatening.

The unique qualities of echocardiography are the time spent on data acquisition and its noninvasive nature. Many investigators attempted to use echocardiography to quantify several

\footnotetext{
* Corresponding author. Rua Lucília Simões, $17,1^{\circ}$ esq., 1500-386, Portugal. Tel.: 35121 7924274; fax: +351 217924271 .

E-mail addresses: p.marcelino@netcabo.pt, pamarcelino@hccabral.min-saude.pt (P.A. Marcelino).
}

physiologic parameters, traditionally obtained by invasive means (noninvasive Swan-Ganz) [1-6]. As echocardiographic determination of pulmonary occlusion pressure suggests, these methods are based on acquisition of fine Doppler studies to apply an equation. The real impact on clinical practice in the ICU is not known. At this stage it is not possible to state that data quantification by bedside echocardiography is suitable in hemodynamic or respiratory unstable patients. Other nonquantitative approaches of echocardiography in the ICU have been described [7-10] in the literature, and these are closer to the current practice of echocardiography in the ICU.

Transesophageal echocardiography (TEE) has gained more attention in the ICU [11-17], because of higher quality images and lower failure rate of examinations performed. 
Transthoracic echocardiography (TTE) is simpler and several descriptions of practical approaches in the ICU are based on this technique $[9,18]$. However, several concerns regarding the ability of data acquisition of TTE in the ICU have been raised [19].

In our ICU, TTE is a routine examination for the last 6 years. Medical staff was trained in this technique, and gained autonomy in performing TTE. Our ICU has a leading position in teaching programs in this field in our country, and currently several protocolized TTE studies are routine.

The present survey addressed two major questions: (1) what sort of data can be obtained using TTE in a general ICU and (2) what is the prevalence of specific cardiac structural and functional abnormalities and diagnoses as well as its influence on mortality and ICU stay.

\section{Materials and methods}

\subsection{Patients}

A TTE was performed in the first $24 \mathrm{~h}$ of admission in the ICU. Regardless of hemodynamic or respiratory conditions, patients were consecutively enrolled during the 18-month period. All patients were characterized according to age, sex, ICU stay, severity scores (Acute physiology and Chronic Heath Evaluation (APACHE II) and Simplified Acute Physiology Score (SAPS II)), and the outcome (ICU mortality).

The following parameters were obtained during the TTE performance: mean arterial pressure (MAP), central venous pressure (CVP) whenever available, and heart rate (HR). These data were obtained by AGILENT monitors, connected to each patient at bedside, using invasive (CVP and mostly MAP) and noninvasive means. For invasive determinations, pressure transducers were zero-referenced at mid-chest.

For general clinical characterization, the following diagnoses were admitted: medical patients, surgical patients, and liver transplant patients.

\subsection{Echocardiography}

A TTE examination was performed on all patients by intensive care physicians with training and extensive experience in echocardiography (4-11 years) in the ICU setting. Whenever needed, a senior cardiology consultant was requested to review recorded examinations. Whenever TTE information was considered inadequate, TEE was performed.

The following parameters were registered: dimensions of cardiac chambers (using the parasternal long and short axis views) and left ventricular (LV) systolic function, which is determined by the shortening the fraction obtained in M-mode using the parasternal long- or short-axis views. An LV shortening fraction was considered normal if higher than $28 \%$, and severely depressed if lower than $15 \%$. Cavities were considered enlarged when found to be larger than: $32 \mathrm{~mm} /$ $\mathrm{m}^{2}$ for left ventricle (LV), $22 \mathrm{~mm} / \mathrm{m}^{2}$ for left atrium (LA), and $14 \mathrm{~mm} / \mathrm{m}^{2}$ for right ventricle. Body surface area was determined by a formula described by Jacobson [20].
The parameters determined by pulsed-wave Doppler were: $E / A$ ratio of transvalvular mitral flow and isovolumetric relaxation time of the left ventricle. These parameters were obtained in a 4-chamber view. When present, tricuspid regurgitation was measured using continuous-wave Doppler.

Continuous wave Doppler and color Doppler analysis were used to detect and evaluate valvular dysfunction (stenosis or regurgitation).

Cardiac output was calculated by the aortic flow/velocity integral (FVI), using pulsed-wave Doppler. The cross-sectional area (CSA) of the aortic valvular annulus was obtained in parasternal long-axis view.

The inferior vena cava (IVC) was evaluated by subcostal view at a distance of $2 \mathrm{~cm}$ from the right atrium, and the maximum and minimum diameters were measured. The IVC index was calculated with the equation: (maximum diameter - minimum diameter $) \times 100 /$ maximum diameter. In all ventilated patients, the IVC index was obtained with a maximum positive end-expiratory pressure (PEEP) of $4 \mathrm{~cm} \mathrm{H}_{2} \mathrm{O}$.

Echocardiographic data were collected to determine the possibility of obtaining or quantifying each individual parameter: cardiac chamber dimensions, LV systolic function, Doppler analysis, and IVC visualization.

Any alteration in TTE examination (alteration in cardiac anatomy or function) was considered an abnormality. It was considered to be a severe condition whenever an emergent therapeutic intervention (tamponade) was needed like; a new diagnostic approach was required (a newly diagnosed severe LV dysfunction, detection of endocarditis); or a cardiology consultation was requested because of a specific cardiac situation (detection of unsuspected valvular heart disease). These situations were considered unsuspected, if they were unknown prior to the examination.

\subsection{Statistical analysis}

All descriptive data are presented as mean, standard deviation, and limits. Comparison between groups was performed using the Student $t$-test, and using Mann-Whitney test if normal distribution of variables could not be found.

A multivariate analysis was performed to establish the influence of echocardiographic abnormalities on mortality or ICU stay. For this purpose, a logistic regression analysis was performed (backward-LR), determining the 95\% confidence interval (CI) and odds ratio. Mortality and ICU stay were considered dependent variables, and echocardiographic data as the independent variables.

The statistical program used was SPSS 13.0 for Windows (SPSS Inc., Chicago, Illinois, 2002).

\section{Results}

\subsection{Patients}

During the study period, 704 patients were enrolled with a mean age of $61.5+/-17.5$ years and a mean ICU stay of $10.6+/-$ 17.1 days. During the TTE examination, 362 (51.4\%) 
Table 1

Main demographic and clinical characteristics of patients.

\begin{tabular}{|c|c|c|c|c|}
\hline & All patients & Medical patients & Surgical patients & Liver transplant patients \\
\hline$n$ & 704 & 426 & 181 & 97 \\
\hline Age (years) & $61.5 \pm 17.5$ & $\begin{array}{l}62.7 \pm 17.1 \\
(18-92)\end{array}$ & $66.6 \pm 17.7$ & $47.1 \pm 12.1$ \\
\hline ICU stay (days) & $10.6 \pm 17.1$ & $\begin{array}{l}12.1 \pm 17.7 \\
(0.4-210)\end{array}$ & $9.7 \pm 22.9$ & $6.9 \pm 7.3$ \\
\hline APACHE II & $22.6 \pm 8.9$ & $\begin{array}{l}24.3 \pm 8.6 \\
(9-45)\end{array}$ & $20.6 \pm 8.5$ & $18.2 \pm 9.3$ \\
\hline SAPS II & $52.7 \pm 20.4$ & $\begin{array}{l}56.6 \pm 18.8 \\
(17-105)\end{array}$ & $48.7 \pm 20.9$ & $41.4 \pm 21.2$ \\
\hline MAP (mm Hg) & $79.8 \pm 21.8$ & $\begin{array}{l}78.1 \pm 21.8 \\
(32-132)\end{array}$ & $78.1 \pm 21.8$ & $89.2 \pm 20.7$ \\
\hline HR (bpm) & $99.6 \pm 19.1$ & $\begin{array}{l}100.7 \pm 20.6 \\
(61-134)\end{array}$ & $98.9 \pm 19.8$ & $96.4 \pm 16.7$ \\
\hline CVP (mm Hg) & $10.6 \pm 4.9$ & $\begin{array}{l}11.4 \pm 4.9 \\
(0-24)\end{array}$ & $9.7 \pm 5$ & $9.2 \pm 4.5$ \\
\hline Mortality & $\begin{array}{l}185 \\
(26.3 \%)\end{array}$ & $\begin{array}{l}134 \\
(31.4 \%)\end{array}$ & $\begin{array}{l}38 \\
(20.9 \%)\end{array}$ & $\begin{array}{l}13 \\
(13.1 \%)\end{array}$ \\
\hline
\end{tabular}

Legend: ICU, Intensive Care Unit; MAP, mean arterial pressure; HR, heart rate; CVP, CVP; mm Hg, millimeters of mercury; bpm, beats per minute.

patients were mechanically ventilated and $143(20.3 \%)$ were hemodynamically unstable (hypotension as cause for admission). Main demographic and clinical characteristics are presented in Table 1. The ICU does not routinely admit patients with acute coronary syndromes. However, 19 patients in the cardiovascular group were presented with acute coronary syndrome and were admitted for ventilator support. A known history of myocardial infarction and valvular heart disease were determined in eight and three patients, respectively. All these 30 patients were diagnosed with predictable changes in TTE.

\subsection{Echocardiography}

Data acquisition possibilities are outlined in Table 2. In four patients, it was not possible to obtain any data (impossible examinations). In 38 patients, cardiac chamber dimensions or LV function could not be quantified. In these patients, only qualitative, subjective information was obtained on such parameters.

Information on cardiac chamber dimensions, LV function, and $\mathrm{CO}$ were obtained in the majority of patients. The major problem was the IVC analysis which was detected in $571(81 \%)$ patients. In surgical patients, IVC evaluation was particularly difficult, because of the presence of surgical bandages.

Table 2

Data acquisition possibilities.

\begin{tabular}{ll}
\hline All patients $(n=704)$ & \\
\hline Cardiac chamber dimensions $(n$ and \%) & $689(97.8 \%)$ \\
Left ventricular function $(n$ and \%) & $670(95.2 \%)$ \\
IVC $(n$ and \%) & $571(81 \%)$ \\
Mitral $E / A$ ( $n$ and \%) & $399(85.9 \%$ of patients in sinus rhythm) \\
IVRT $(n$ and \%) & $569(80.9 \%)$ \\
Tricuspid regurgitant jet $(n$ and \%) & $291(41.3 \%)$ \\
Cardiac output $(n$ and \%) & $610(86.7 \%)$ \\
\hline
\end{tabular}

Legend: IVC, inferior vena cava; IVRT, isovolumetric relaxation time.
Although a regurgitant tricuspid jet was detected in $41.5 \%$ of patients, a peak gradient $>40 \mathrm{~mm} \mathrm{Hg}$ was obtained only in 24 of these patients.

At least one anatomic or functional cardiac alteration was found in $234(33 \%)$ patients (Table 3). The most frequent was left atrium enlargement $(n=163)$, followed by LV systolic dysfunction $(n=132)$. Patients with these alterations were older; presented higher APACHE II score and higher mortality; and were admitted with higher CVP (Table 4). Medical patients presented the highest prevalence of TTE alterations (Table 5).

Severe previously unknown situations were detected in 53 (7.5\%) patients (Table 3), representing newly diagnosed clinical conditions. The most frequent condition was severe LV systolic dysfunction which is found in 23 patients. These situations were not linked to acute myocardial ischemia. Severe valvular disease was detected in ten patients (4 severe aortic stenosis, 4 severe aortic regurgitation, and 2 severe mitral regurgitation),

Table 3

Echocardiographic abnormalities detected.

\begin{tabular}{|c|c|c|c|}
\hline \multicolumn{2}{|l|}{ TTE alterations } & \multicolumn{2}{|l|}{$\begin{array}{l}\text { Severe previously unknown } \\
\text { TTE diagnoses }\end{array}$} \\
\hline $\begin{array}{l}\text { Left ventricular } \\
\text { enlargement }(n)\end{array}$ & 65 & $\begin{array}{l}\text { Severe left ventricular } \\
\text { systolic dysfunction }\end{array}$ & 23 \\
\hline $\begin{array}{l}\text { Right ventricular } \\
\quad \text { enlargement }(n)\end{array}$ & 99 & Severe valvular disease & 10 \\
\hline $\begin{array}{l}\text { Left atrium } \\
\quad \text { enlargement }(n)\end{array}$ & 163 & Tamponade & 4 \\
\hline $\begin{array}{l}\text { Left ventricular systolic } \\
\text { dysfunction }(n)\end{array}$ & 132 & Endocarditis & 8 \\
\hline \multirow{3}{*}{ LV hypertrophy $(n)$} & 41 & Others: & \\
\hline & & $\begin{array}{l}\text { Hypertrophic } \\
\text { cardiomyopathy }\end{array}$ & 2 \\
\hline & & Intracardiac mass & 6 \\
\hline Pericardial effusion $(n)$ & 15 & & \\
\hline Total $(n$ and $\%)$ & $234(33 \%)^{\mathrm{a}}$ & & $53(7.5 \%)$ \\
\hline
\end{tabular}

${ }^{\text {a }}$ Note: Many patients presented more than one echocardiographic alteration, thus, the number of patients with these alterations does not correspond to the sum of all alterations. 
Table 4

Differences between patients with and without echocardiographic alterations.

\begin{tabular}{llll}
\hline Parameter & $\begin{array}{l}\text { Patients without } \\
\text { echocardiographic } \\
\text { alterations }\end{array}$ & $\begin{array}{l}\text { Patients with } \\
\text { echocardiographic } \\
\text { alterations }\end{array}$ & $p$ value \\
\hline$N$ & 470 & 234 & \\
Age (years) & $58.1 \pm 17.4(21-92)$ & $66 \pm 16.5(18-92)$ & $<0.001$ \\
ICU stay (days) & $10.9 \pm 18.4(0.4-119.3)$ & $10.9 \pm 16.5(0.4-177)$ & $\mathrm{ns}$ \\
APACHE II & $21.1 \pm 8.9(9-45)$ & $24.4 \pm 8.7(9-47)$ & $<0.001$ \\
SAPS II & $48.7 \pm 21(9-115)$ & $57.4 \pm 18.8(27-105)$ & $\mathrm{ns}$ \\
Mortality & $84(25.4 \%)$ & $94(40.1 \%)$ & $<0.001$ \\
MAP (mm Hg) & $82.7 \pm 20.9(32-141)$ & $77.2 \pm 22(35-134)$ & $<0.001$ \\
HR (bpm) & $99.6 \pm 18.8(66-137)$ & $99.4 \pm 20.8(63-134)$ & $\mathrm{ns}$ \\
CVP (mm Hg) & $9.4 \pm 4.6(-2$ to 22$)$ & $12.4 \pm 4.8(4-27)$ & $<0.001$ \\
IVC maximum & $14.7 \pm 4.4(5-30)$ & $18.3 \pm 4.6(9-28)$ & $<0.001$ \\
$\quad$ dimension (mm) & & & $<0.001$ \\
IVC index (\%) & $33 \pm 26(0-100)$ & $23 \pm 23(0-100)$ & $<0.001$ \\
Cardiac index & $3.89 \pm 0.93$ & $3.34 \pm 0.95(1.3-6.97)$ &
\end{tabular}

Legend: ICU. Intensive Care Unit; MAP, mean arterial pressure; HR, heart rate; CVP, central venous pressure; IVC, inferior vena cava; $1 / \mathrm{min} / \mathrm{m}^{2}$, liters per minute per squared meter; $\mathrm{mm} \mathrm{Hg}$, millimeters of mercury; ns, nonsignificant.

and a hypertrophic cardiomyopathy was diagnosed in two patients. To review the recorded TTE, a cardiology consultation was requested in 14 cases; a new diagnosis was established in one case. The TEE was used in 22 cases because of inadequate TTE information.

Through multivariate analysis, tricuspide regurgitation correlated independently with mortality $(p=0.016$, Confidence Interval 1.007-1.067). Mortality was also independently affected by ICU stay (CI 1.0-1.019, $p<0.001$ ). Each day of ICU stay increased mortality in $1.9 \%$ of the patients.

\section{Discussion}

Routine use of TTE by all staff physicians in a general ICU is not a common finding. In this survey we presented and evaluated this practice, making possible to assess important issues like data acquisition possibilities of TTE and the prevalence of specific cardiac abnormalities or diagnoses.

In the ICU, TTE examination was reported to present a high failure rate. A study by Cook et al. [19] observed a failure of $38 \%$ in a surgical ICU, where TTE examinations were considered "inadequate" or "poor." These authors used TEE as a comparative examination. Conditions related to failure were identified and were found to be related to edema (weight gain $>10 \%$ from the baseline), presence of chest tube thoracostomies, and high positive end expiratory pressures (PEEP $>15 \mathrm{~mm} \mathrm{Hg}$ ). The LV function could be evaluated in 65 (64\%) patients, but even diagnoses easily assessed by TTE, like tamponade, had a high failure rate. This failure rate described by Cook et al. seemed to link to specific situations such as those described (presence of chest devices) and encountered mainly in surgical patients. In this study, there were difficulties in evaluating surgical patients. Because patients with chest tubes thoracostomies were few, the main difficulty observed in surgical patients was detecting IVC.
Another study by Stanko et al. [21], which was performed in a medical or surgical ICU, observed a lower rate (13 of 135) of "suboptimal studies." Bossone et al. [22] described 2 of the 467 consecutive studies in which the TTE examinations were impossible to interpret.

This study was performed in a general ICU, where most patients presented a medical clinical condition. It seemed that the characteristics of ICU patients are relevant in defining the best echocardiographic tool (TTE or TEE).

In addition, the information needed may be also important. It is well recognized that TEE is superior to TTE in identifying a valvular disease (like in cardiac surgery patients) or a cardiac source of embolus [11,14,23].

The detecting capability of several Doppler variables was evaluated in this study. The authors could not find similar data in the literature, but would like to emphasize some difficulties in obtaining these data. Mitral $E / A$ was analyzed in $85.9 \%$ of patients in sinus rhythm. However, the mean heart rate of patients evaluated was high and a fusion of waves was frequently observed. The authors suspect that several other Doppler-derived parameters such as the deceleration time or acceleration time would be more difficult to obtain. Other important Doppler analyses were not carried out including the determination of flow propagation velocity. Overall, Doppler analysis could have a higher failure rate if a thorough examination was performed. This issue may be important. A considerable number of equations, especially those aimed to quantify pulmonary wedge pressure, rely on Doppler determination of several variables.

A tricuspid regurgitant jet was detected in $41.3 \%$ of patients studied, a lower rate than described by Borgeson et al. [24]. This may be related to the fact that most patients did not present pulmonary hypertension. Cardiac output determination may present a different condition. This parameter may be important in determining hemodynamic profiles, particularly in hypotensive patients (a normal or high $\mathrm{CO}$ in hypotensive patients is usually a result of low systemic vascular resistance). The high rate of detection observed allows us to establish this parameter as a routine evaluation in the ICU. For CO determination, a pulsed-wave Doppler analysis of LV outflow was used. This method has proved to correlate well to thermodilution technique $[25,26]$.

The second issue addressed was related to a routine use of TTE. This question covers several topics such as the detection and study of the prevalence of cardiac abnormalities in a general ICU, detection of specific diagnoses, and the influence of these on the outcome.

Table 5

Prevalence of echocardiographic alterations in different diagnostic groups.

\begin{tabular}{lrc}
\hline & $n$ & $\begin{array}{l}\text { Detected echocardiographic alterations } \\
(n \text { and \%) }\end{array}$ \\
\hline All patients & 704 & $234(33.2 \%)$ \\
Medical patients & 426 & $191(44.8 \%)$ \\
Surgical patients & 181 & $45(24.8 \%)$ \\
Liver transplant patients & 97 & $8(8.2 \%)$ \\
\hline
\end{tabular}


In the study by Stanko et al. [21], a high number of abnormalities were detected (121 of 135). The main difference between these studies lies in the methodology used. Stanko et al. enrolled patients on the basis of a specific request for TTE (selective TTE), whereas this study enrolled consecutive patients. It is possible that patients enrolled by those authors had a higher previous suspicion for cardiovascular abnormalities.

In the other study by Bossone et al. [22], the rate of abnormalities was $36 \%$ (169 of 467 patients), similar to the prevalence rate of abnormalities detected in the present study. Also, a number of severe conditions that required unblindedness of the study were detected in $11 \%$ (52 of 467 patients). It should be noted that Bossone et al. excluded patients with "obvious cardiovascular disease" which was determined on admission. Differences between these studies should be pointed out. Bossone et al. prospectively defined the abnormalities encountered, considering all abnormalities, LV dysfunction, and severe chamber dilatation, regardless of its clinical significance. In this study, TTE abnormalities were differentially analyzed; it included any anatomic or functional alteration and specific severe cardiac diagnoses.

Right heart chamber dilatation and signs of RV overload were not identified in this study, but they were mostly present in patients with respiratory diseases. However, in a patient's assessment, this information is relevant.

In this study we observed that tricuspid regurgitation influenced mortality ( $p=0.016$, CI $1.007-1.067)$, by means of multivariate analysis. Swanchuk et al. [27] also described similar data. They studied 942 patients using TTE performed up to 6 months after discharge from the ICU, and describe a worse outcome in patients with LV dysfunction, with severe tricuspid and aortic regurgitation as well as, signs of pulmonary hypertension. Our data should be carefully taken into account as it can be affected by several parameters, such as, characteristics of studied patients, and study design. More importantly a tricuspid regurgitant jet was detected in $293(41.3 \%)$ of the 703 patients. Taking this into consideration it is not possible to generalize these findings to all ICU patients.

Finally, in addition to the information on the feasibility of bedside TTE in the ICU, the study can contribute to establish the training required for ICU physicians. It should be remembered, however, that such training may vary according to patients' characteristics and ICU needs.

\subsection{Study limitations}

Several limitations must be pointed out. First, this was an observational rather than a blinded study. Only patients with known diagnoses, previous to admission, were considered to have suspected cardiac abnormalities. Furthermore, all examinations were performed by ICU physicians, and the information was immediately available. Hence, other diagnostic tests were not considered; we could not establish the clinical suspicion for more severe conditions. But even in this case, TTE allowed a time-to-diagnosis gain.

We found a significant number of patients with several echocardiographic disorders. It is possible to state that the real impact of echocardiography in major outcomes, such as mortality, is yet to be established. We are not aware of any study addressing this issue.

\subsection{Conclusion}

The TTE is a useful technique in a general ICU. Most echocardiographic parameters can be obtained and quantified. Specific cardiac abnormalities, structural or functional, were detected in $33 \%$ of the patients. The LA enlargement and LV systolic dysfunction were the more frequently detected abnormality. As much as $7.5 \%$ of the patients presented previously unknown critical conditions. These were represented by severe structural cardiac diseases that could threaten the patient's life.

In the cohort studied, mortality was independently influenced by tricuspid regurgitation $(p=0.116$, CI $1.007-1.067)$.

\section{Learning points}

- Echocardiography is a promising technique in the Intensive Care. Internal Medicine specialists can perform this technique accurately after appropriate training in several non cardiological settings such as Intensive Care Units (ICU).

- This study demonstrates that the transthoracic approach can provide information on cardiac anatomy and function in most ICU patients, as well as detect severe previously unknown conditions in some patients.

\section{References}

[1] Appleton CP, Galloway JM, Gonzalez MS, Basnight MA. Estimation of left ventricular filling pressures using two-dimensional and Doppler echocardiography in adult patients with cardiac disease. Additional value of analysing left atrial size, left atrial ejection fraction and the difference in duration of pulmonary venous and mitral flow velocity at atrial contraction. J Am Coll Cardiol 1993;22:1972-82.

[2] Chirillo F, Brunazzi MC, Barbiero M, Giavarine D, Pasquelini M, Francheschini-Grisolia E, et al. Estimating mean pulmonary wedge pressure in patients with chronic atrial fibrillation from transthoracic Doppler indexes of mitral and pulmonary venous flow velocity. J Am Coll Cardiol 1997;30:19-26.

[3] Gonzalez-Vilchez F, Ares M, Ayuela J, Alonso L. Combined use of pulsed and color M-mode Doppler echocardiography for the estimation of pulmonary capillary wedge pressure: an empirical approach based on an analytical relation. J Am Coll Cardiol 1999;34:515-23.

[4] Nagueh SF, Kopelen HN, Zoghbi WA. Feasibility and accuracy of Doppler echocardiographic estimation of pulmonary artery occlusive pressure in the intensive care unit. Am J Cardiol 1995;75:1256-62.

[5] Nagueh SF, Middleton KJ, Kopelen HN, Zoghbi WA, Quinones MA. Doppler tissue imaging: a noninvasive technique for evaluation of left ventricular relaxation and estimation of pressures. J Am Coll Cardiol 1997;30:1527-33.

[6] Ommen S, Nishimura RA, Appleton CP, Miller FA, Oh JK, Redfield MM, Tajik AJ. Clinical utility of Doppler echocardiography and tissue Doppler imaging in the estimation of left ventricular filling pressures. Circulation 2000;102:1788-94.

[7] Kaul S, Stratienko AA, Pollock SG, Marieb MA, Keller MW, Sabia PJ. Value of two-dimensional echocardiography for determining the basis of hemodynamic compromise in critically ill patients: a prospective study. J Am Soc Echocardiogr 1994;7:598-606. 
[8] Jensen MB, Sloth E, Larsen KM, Schmidt MB. Transthoracic echocardiography for cardiopulmonary monitoring in intensive care. Eur $\mathrm{J}$ Anaesthesiol 2005;21:700-7.

[9] Joseph MX, Disney PJS, Da Costa R, et al. Transthoracic echocardiography to identify or exclude cardiac cause of shock. Chest 2004;126:1592-7.

[10] Vieillard-Baron A, Prin S, Chergui K, Dubourg O, Jardin F. Hemodynamic instability in sepsis: bedside assessment by echocardiography. Am J Resp Crit Care Med 2003;168:1270-6.

[11] Cicek S, Demirilic U, Kuralay E, Tatar H, Ozturk O. Transesophageal echocardiography in cardiac surgical emergencies. J Card Surg 1995; 10:236-44.

[12] Descorps-Declerc A, Smail N, Vigue B, Davanteau J, Mimoz O, Edouard A, Samii K. Transgastric, pulsed Doppler echocardiographic determination of cardiac output. Intensive Care Med 1996;22:34-8.

[13] Heidenreich PA, Stainback RF, Redberg RF, Schiller NB, Cohen NH, Foster E. Transesophageal echocardiography predicts mortality in critically ill patients with unexplained hypotension. J Am Coll Cardiol 1995;26:152-8

[14] Huttemann E. Transesophageal echocardiography in critical care. Minerva Anestesiol 2006;15:345-9.

[15] Kuhl HP, Hanrath P. The impact of transesophageal echocardiography on daily clinical practice. Eur J Echocardiogr 2004;5:455-68.

[16] Marik P. Pulmonary artery catheterization and esophageal Doppler monitoring in the ICU. Chest 1999;116:1085-91.

[17] Milani RV, Lavie CJ, Gilliland YE, Cassidy MM, Bernal JA. Overview of transesophageal echocardiography for chest physician. Chest 2003;124:1081-9.
[18] Jensen MB, Sloth E, Larsen KM, Schmidt MB. Transthoracic echocardiography for cardiopulmonary monitoring in intensive care. Eur J Anaesthesiol 2004;21:700-7.

[19] Cook CH, Praba AC, Beery PR, Martin LC. Transthoracic echocardiography is not cost-effective in critically ill surgical patients. J Trauma 2002;52:280-4.

[20] Marino Paul L. The ICU book. 2nd ed. Baltimore, Maryland: William \& Wilkins; 1998.

[21] Stanko LK, Jacobshn E, Tam JW, De Wet CJ, Avidan M. Transthoracic echocardiography: impact on diagnosis and management in tertiary care intensive care units. Anaesth Intensive Care 2005;33:492-6.

[22] Bossone E, DiGiovine B, Watts S, Markovitz PA, Carey L, Watts C, Armstrong W. Range and prevalence of cardiac abnormalities in patients hospitalized in a medical ICU. Chest 2002;122:1370-6.

[23] Gan TJ. The esophageal Doppler as an alternative to the pulmonary artery catheter. Curr Opin Crit Care Med 2000;6:214-21.

[24] Borgeson DD, Seward JB, Miller Jr FA, Oh JK, Tajik AK. Frequency of Doppler measurable pulmonary artery pressures. J Am Soc Echocardiogr 1996;9:832-7.

[25] McLean AS. Echocardiography in the Intensive Care Unit. Intensive Care World 1996;13:12-7.

[26] Shuster AH, Nanda NC. Doppler echocardiography measurement of cardiac output: comparison with a non golden standard. Am J Cardiol 1984;53:257-61.

[27] Swanchuk CW, Wong DT, Kavanagh BP, Siu SC. Transthoracic echocardiography does not improve prediction of outcome over APACHE II in medical-surgical intensive care. Can J Anaesth 2003;50:305-10. 